\title{
Potential diagnostic value of miR-155 in serum from lung adenocarcinoma patients
}

\author{
FENG GAO, JINGXIA CHANG, HUAQI WANG and GUOJUN ZHANG \\ Division of Respiratory and Critical Care Medicine, The First Affiliated Hospital \\ of Zhengzhou University, Zhengzhou, Henan 450052, P.R. China
}

Received September 11, 2013; Accepted October 22, 2013

DOI: $10.3892 /$ or.2013.2830

\begin{abstract}
Recent studies have demonstrated that microRNAs (miRNAs) are stably detectable in plasma/serum. The expression profile of miR-21 and miR-155 was evaluated in the present study, since miR-21 is frequently reported as highly expressed in several types of cancers, while miR-155 was also found to be significantly expressed in lung cancer cell lines. Using in vitro studies, we found that miR-155 could be a candidate plasmatic biomarker for diagnosing lung cancer. We assessed the differences in levels of miR-21, miR-155, carcinoembryonic antigen (CEA) and carbohydrate antigen 125 (CA-125) expression in serum samples between lung cancer patients and healthy controls. We estimating the clinical diagnostic value of miR-155 independently and combined with CA-125 and/ or CEA levels. The present study consisted of three parts: i) confirmation of the stable expression of miR-155 in the patient serum samples using quantitative PCR; ii) confirmation of higher miR-155, CEA and CA-125 levels in the patient serum samples when compared with levels in the normal controls by quantitative PCR; iii) evaluation of miR-155, CEA and CA-125 concentrations in serum sampes for tumor diagnosis of lung adenocarcinoma via ROC (receiver-operating characteristic) analysis. The results showed that i) expression of miR-155 was significantly higher in the serum of lung adenocarcinoma patients than that in normal controls $(\mathrm{P}<0.05)$; ii) testing results of serum miR-155 levels showed a much higher sensitivity (0.722) than that for CA-125 or CEA; iii) CEA associated with CA-125 had the highest Youden's index (0.639) in all terms of combinations; and iv) combined with CA-125 testing, miR-155 received a competitive sensitivity (0.889) and specificity (0.688) for diagnosing lung adenocarcinoma $(\mathrm{OOP}=14.88)$. In conclusion, endogenous miR-155 stably existed in patient serum and could be sensitively and specifically measured. Overexpression
\end{abstract}

Correspondence to: Professor Guojun Zhang, Division of Respiratory and Critical Care Medicine, The First Affiliated Hospital of Zhengzhou University, 1 Jianshe Road, Zhengzhou, Henan 450052, P.R. China

E-mail: zhgjzz@gmail.com

Key words: lung adenocarcinoma, miR-155, biomarker, serum of miR-155 in serum specimens could constitute a diagnostic marker for the early detection of lung adenocarcinoma.

\section{Introduction}

Lung cancer is one of the most leading causes of cancer-related mortality worldwide. Various strategies have been adopted for the management of this disease. Adenocarcinoma, located in the mucus cells within airways, is a main type of lung cancer, and accounts for $\sim 40 \%$ of all lung cancers and is often diagnosed at a late stage with concomitant poor prognosis (1).

Although tumor biomarkers have greatly improved the diagnosis of this disease, the invasive, unpleasant and inconvenient nature of current diagnostic procedures limit their application (2). Hence, there is an urgent need for identification of novel non-invasive biomarkers for early tumor detection. microRNAs (miRNAs), a class of naturally occurring small non-coding RNAs, usually 18-25 nucleotides (18-25 nt) in length, have been linked to cancer development. Recently, altered miRNA expression has been reported in various types of cancers, and the profiles of tissue miRNAs exhibit great potential for application in cancer diagnosis $(3,4)$.

In the present study, we demonstrated that a specific miRNA, hsa-miR-155 (miR-155), shows great promise as a novel non-invasive biomarker in serum for the diagnosis of adenocarcinoma lung cancer. To validate whether altered levels of miR-155 expression detected in serum could be a useful biomarker for the diagnosis of NSCLC (non-small cell lung cancer), particularly in adenocarcinoma, real-time relative quantitative reverse transcription-polymerase chain reaction (RT-PCR) was performed. The results were associated with ROC (receiver-operating characteristic) curve analysis in both the patient and control cohorts in order to ascertain i) whether miR-155 is able to be reliably measured in serum, ii) whether there are any significant differences between patients and controls regarding miR-155 expression in serum, and iii) whether analysis of miR-155 expression in serum may faciliate the diagnosis of lung adenocarcinoma.

\section{Materials and methods}

Origin of the serum samples. All serum samples used in the present study were collected at the Divison of Respiratory and Critical Care Medicine, The First Affiliated Hospital 
Table I. Demographic information of the lung adenocarcinoma cases and controls.

\begin{tabular}{lccc}
\hline & $\begin{array}{c}\text { Patients } \\
(\mathrm{n}=36)\end{array}$ & $\begin{array}{c}\text { Controls } \\
(\mathrm{n}=32)\end{array}$ & P-value \\
\hline $\begin{array}{l}\text { Mean age (years) } \\
\text { Gender }\end{array}$ & 55.1 (SD 12.2) & 52.5 (SD 11.3) & $0.011^{\mathrm{a}}$ \\
Female & 25 & 22 & $0.003^{\mathrm{a}}$ \\
Male & 11 & 10 & \\
Smoking status & & & \\
Packs/year & $36.8(\mathrm{SD} 12.6)$ & 0 & \\
Location of tumor & & & \\
Central & 8 & & \\
Peripheral & 28 & & \\
Cancer stage & & & \\
I & 6 & & \\
II & 8 & & \\
III & 15 & & \\
IV & 7 & & \\
Histology & & & \\
AC of lung & 36 & & \\
SC of lung & 0 & & \\
& & & \\
& & & \\
\end{tabular}

AC, adenocarcinoma; SC, squamous cell carcinoma. ${ }^{\text {aC Compared }}$ between NSCLC and control groups; ${ }^{b}$ compared within NSCLC group.

of Zhengzhou University. Samples were obained at both the out-patient and in-patient departments from January 2012 to January 2013. The serum samples were obtained from 36 patients with lung cancer and 32 healthy volunteers whose age and gender distributions showed no differences with the patients.

All patients with lung cancer were pathologically diagnosed, were diagnosed with lung cancer for the first time, and did not receive prior chemotherapy, radiotherapy or other tumor-specific treatment. To control disease heterogeneity, lung adenocarcinoma patients at all stages from I to IV were involved in the present study. Patients known to have a family history of cancer or metastatic cancer from other or unknown origins were excluded. All controls had no family history or past history of cancer. All controls were non-smokers (Table I).

Serum collection and RNA extraction. Whole blood $(2 \mathrm{ml})$ from every patient and negative control was collected in regular tubes and immediately processed to prevent contamination by cellular nucleic acids. Blood samples were centrifuged at 2,000 rpm for $10 \mathrm{~min}$ at room temperature, and then the upper supernatant, which was the serum sample, was transferred to new RNase-free collection tubes, respectively, and stored at $-80^{\circ} \mathrm{C}$ for further processing.

Small RNA molecules with size $<200$ nucleotides were extracted and purified from $200 \mu \mathrm{l}$ of each serum sample, using an E.Z.N.A. ${ }^{\circledR}$ miRNA kit (Omega Bio-Tek, Inc., Norcross, GA,
Table II. miRNA real-time-PCR primer sequences.

\begin{tabular}{ll}
\hline Primer & \multicolumn{1}{c}{ Sequence (5'-3') } \\
\hline U6 forward & CTCGCTTCGGCAGCACA \\
U6 reverse & AACGCTTCACGAATTTGCGT \\
miR-21 forward & TAGCTTATCAGACTGATGTTGA \\
miR-155 forward & TTAATGCTAATCGTGATAGGGGT \\
Universal reverse & GTGCAGGGTCCGAGGT \\
\hline
\end{tabular}

USA) and finally eluted into $15 \mu \mathrm{l}$ of Elution solution according to the manufacturer's instructions.

Reverse transcription and real-time RT-PCR. RNA was applied for RT by using the Veriti ${ }^{\mathrm{TM}}$ 96-Well Thermal Cycler (Applied Biosystems, Foster City, CA, USA) and Roche ${ }^{\circledR}$ Transcriptor First-Strand cDNA Synthesis kit (F. Hoffmann-La Roche Ltd.), according to the manufacturer's instructions. The reaction included $1 \mu \mathrm{g}$ total RNA, $2 \mu \mathrm{l}$ random hexamer primer at $60 \mu \mathrm{M}, 4 \mu \mathrm{l} 1 \mathrm{X}$ RT reaction buffer, $1 \mathrm{mM}$ of each dNTP, $10 \mathrm{U}$ MultiScribe reverse transcriptase and PCR-grade water to make a total volume of $20 \mu \mathrm{l}$. The RT reaction was incubated for $10 \mathrm{~min}$ at $25^{\circ} \mathrm{C}$, followed by $30 \mathrm{~min}$ at $55^{\circ} \mathrm{C}$, and then the transcriptor reverse transcriptase was inactivated by heating at $85^{\circ} \mathrm{C}$ for $5 \mathrm{~min}$. The reaction was stopped by placing the tubes on ice for further use.

Real-time PCR was performed to measure the expression levels of target miRNAs, using Roche ${ }^{\circledR}$ FastStart Universal SYBR-Green Master (ROX) PCR kit (F. Hoffmann-La Roche Ltd.) on the 7500 Fast Real-time PCR system (Applied Biosystems). The $20-\mu 1$ PCR reaction included RT products, Roche ${ }^{\circledR}$ ROX and the corresponding primers for the target genes. The reactions were incubated in a $96-$ well plate at $95^{\circ} \mathrm{C}$ for $10 \mathrm{~min}$, followed by 40 cycles of $95^{\circ} \mathrm{C}$ for $10 \mathrm{sec}$ and $58^{\circ} \mathrm{C}$ for $30 \mathrm{sec}$. The threshold cycle $(\mathrm{Ct})$ was defined as the fractional cycle number at which the fluorescence passed the fixed threshold. Expression levels of hsa-miR-21 and hsa-miR155 were normalized in relation to the expression of U6 RNA, a small nuclear RNA as an internal control commonly used for miRNA quantification assay (5). The primer sequences for miRNA RT-PCR are shown as Table II. All assays were performed in triplicate.

Detection of CEA and CA-125. Values for carcinoembryonic antigen (CEA) and carbohydrate antigen 125 (CA-125) levels in the serum of the lung adenocarcinoma patients and controls were determined at the Clinical Laboratory of The First Affiliated Hospital of Zhengzhou University.

Statistical analysis. Expression levels of the miRNAs were calculated by cycle threshold $(\mathrm{Ct})$ values with SDS 2.0 software (Applied Biosystems). The concentrations from serum samples were normalized using the $2^{-\Delta \Delta \mathrm{Ct}}$ method relative to U6 small nuclear RNA (RNU6B) (6). The value of $\Delta \mathrm{Ct}$ was calculated by subtracting the $\mathrm{Ct}$ values of RNU6B from the $\mathrm{Ct}$ values of the miRNAs of interest in the study. The values of $\Delta \Delta \mathrm{Ct}$ were then calculated by subtracting the $\Delta \mathrm{Ct}$ of the control 


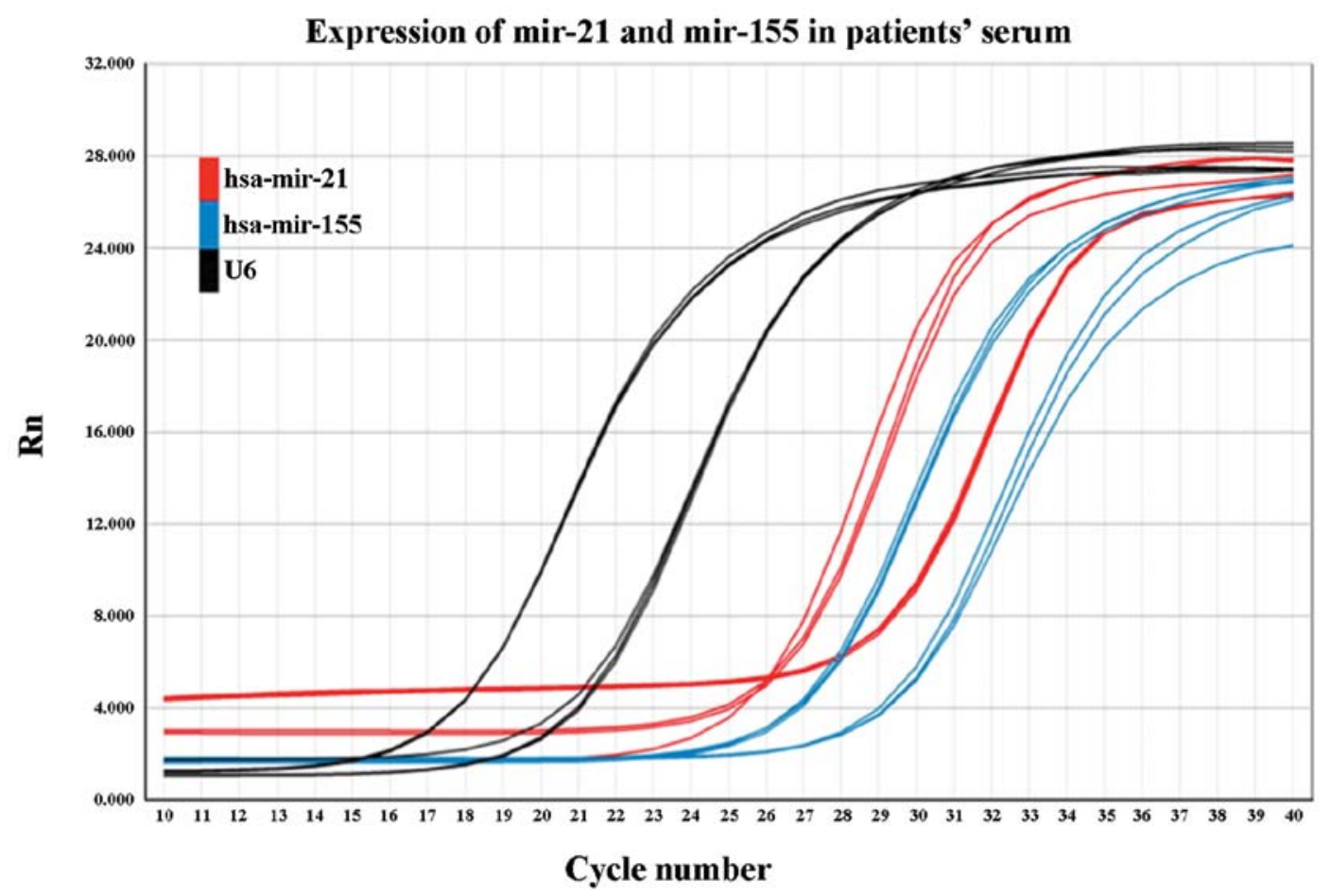

Figure 1. RT-PCR amplication cruve of miR-21 and miR-155 expression in the serum of lung adenocarcinoma patients.

samples from the $\Delta \mathrm{Ct}$ of the cancer patients. The change in gene expression was calculated using the equation $2^{-\Delta \Delta \mathrm{Ct}}(7)$.

The independent-samples t-test for unpaired data was performed to compare differences in miRNA concentrations in serum between cancer patients and healthy volunteers. A $\mathrm{P}$-value $<0.05$ was considered to indicate a statistically significant result.

Receiver-operating characteristic (ROC) curves and the area under the ROC curve (AUC) were used to assess the feasibility of serum miRNA as a diagnostic tool for detecting lung adenocarcinoma (8). The values for specificity, sensitivity, positive/negative likelihood ratio and Youden's index for each factor and the combined groups were used to determine the cut-off value for the serum miR-155 concentrations with which to distinguish the patients and healthy controls. All results are expressed as means $\pm \mathrm{SD}$

\section{Results}

miR-21 and miR-155 expression in serum of adenocarcinoma patients and healthy control. All samples were tested at least four times, and there were triple repeat contrasts for each sample every time RT-PCR was run. Results showed that the studied miRNAs could be stably detected in the serum samples from both the patients and healthy controls. However, low levels of miR-21 and miR-155 expression were noted in the control group, while U6, as an internal reference, was easily amplified at high levels (Fig. 1).

miR-21 and miR-155 were assessed in both control and patient groups. $2^{-\Delta \mathrm{Ct}}$ was the relative expression value while the fold-difference was determined after defining miR- $155_{\text {control }}$ as the reference. In that case, the fold-difference of miR$155_{\text {control }}$ was 1 , and the miR- $21_{\text {control }}$ was 1.173 , which indicated that the expression of miR-21 in the control group was 1.173 times that of the mean level of miR-155; the fold-difference of miR-155 patient was 2.034 and miR-21 $1_{\text {patient }}$ was 5.546, which indicated levels were 2.034 times and 5.546 times higher when compared with the expression levels of miR-155 $5_{\text {control }}(\mathrm{P}<0.05)$. In general, compared with the patients and control groups, there were noticeable differences in miR-21 and miR-155 expression, respectively between the two groups. Within the patient group, significant differences were also found between miR-21 and miR-155 expression $(\mathrm{P}<0.05)$ (Table III; Fig. 2).

CEA and CA-125 expression in serum of adenocarcinoma patients and healthy controls. The difference in CEA and CA-125 expression between the control and patient groups was significant. Thus we assessed levels of these two factors in the present study, and analyzed their value when combined with miR-155 expression. All CEA and CA-125 tests were carried out at the Clinical Laboratory of our hospital, and the results are shown in Table IV.

ROC analysis to evaluate diagnostic efficiency. Receiveroperating characteristic analysis of CEA, CA-125 and miR-155 was performed with SPSS 20.0, and the curve and results are shown in Fig. 3 and Table V. Regarding the area under the ROC curve, CA-125 had the highest value at 0.816 and the value for miR-155 was 0.761, while CEA had the lowest value of the three at 0.712 . Based on these results, further analysis with multiple indices was carried out to evaluate their diagnostic value, respectively, and in combination with each other.

For purposes of estimation, the sensitivity and specificity values are better at high levels. Moreover, it is the same when Youden's index is used. The positive likelihood ratio (+LR) indicates the probability for a correct diagnosis; higher values 
Table III. Relative expression levels of miR-21 and miR-155 in the serum samples of the lung adenocarcinoma patients and controls.

\begin{tabular}{llllcrcr}
\hline Groups & miRNA & Ct value & U6 Ct value & $\Delta \mathrm{Ct}$ & $\Delta \Delta \mathrm{Ct}$ & $2^{-\Delta \mathrm{Ct} \times 10^{3}}$ & Fold-difference $\left(2^{-\Delta \Delta \mathrm{Ct})}\right.$ \\
\hline Control & miR-155 & $32.904 \pm 0.42$ & $21.423 \pm 0.57$ & $11.480 \pm 0.51$ & $0.000 \pm 0.01$ & $1.028 \pm 0.124$ & $0.999 \pm 0.01$ \\
& miR-21 & $32.938 \pm 0.65$ & & $11.514 \pm 0.58$ & $0.0337 \pm 0.57$ & $1.260 \pm 0.15$ & $1.173 \pm 0.54$ \\
Patient & miR-155 & $30.276 \pm 0.62$ & $20.914 \pm 0.55$ & $9.362 \pm 0.69$ & $-2.125 \pm 0.88$ & $2.074 \pm 0.81$ & $2.034 \pm 2.74^{\mathrm{a}, \mathrm{b}}$ \\
& miR-21 & $28.805 \pm 0.61$ & & $7.891 \pm 0.67$ & $-3.597 \pm 0.80$ & $5.696 \pm 1.89$ & $5.546 \pm 6.43^{\mathrm{a}, \mathrm{b}, \mathrm{c}}$ \\
\hline
\end{tabular}

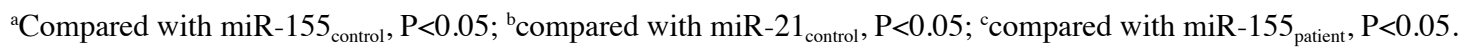

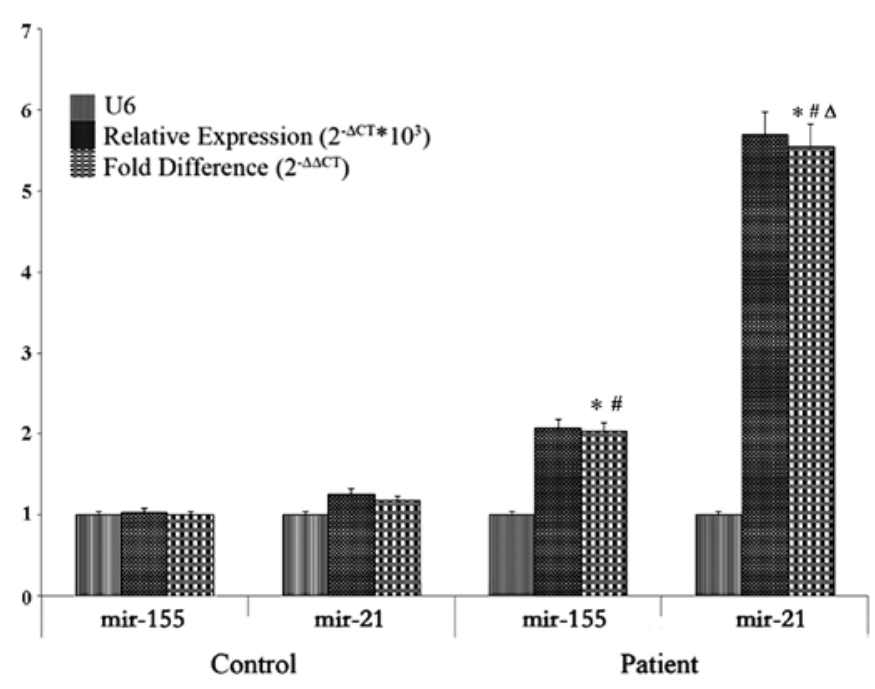

Figure 2. Relative expression of miR-21 and miR-155 in serum samples of the lung cancer patients and healthy controls. * Compared with miR-155 $5_{\text {control }}$, $\mathrm{P}<0.05 ;$ " ${ }^{\prime}$ compared with miR-21 $1_{\text {control }}, \mathrm{P}<0.05 ;{ }^{\Delta}$ compared with miR-155 $5_{\text {patient }}$, $\mathrm{P}<0.05$.

are optimal; a value that approaches 10 is regarded as a perfect suitable condition. The negative likelihood ratio (-LR) is the indicator for the probability of a missed diagnosis; a lower value is optimal.

In the present study, of all the three independent assays for CEA, CA-125 and miR-155 each, miR-155 had the best sensitivity and CA-125 had the best specificity, LRs and YI. In regards to the combined group analysis, CEA + CA-125 had the best score for specificity, LRs and YI. When we combined CEA or CA-125 with miR-155, the sensitivity of each group was highly improved when compared with observation of the single marker alone. Moreover, the CA-125 + miR-155 group had higher sensitivity, -LR and YI than these values for the CEA + miR-155 group (Table VI).

\section{Discussion}

miRNAs, as a class of small non-coding RNAs, have been found to be involved in cancer initiation and progression. During the last decade, researchers have focused on the expression profiles of types of miRNAs that may be used for classification, diagnosis and prognosis of human malignancies (9).

The fact that more than $50 \%$ of miRNAs are located at cancer-related chromosomal regions supports the idea that
Table IV. Expression levels of CEA, CA-125, miR-155 in the serum sampes of the lung adenocarcinoma patients and controls.

\begin{tabular}{lllllll}
\hline & Groups & N & Mean & SD & SEM & P-value \\
\hline CEA & Patient & 36 & 30.456 & 18.464 & 3.077 & \\
& Control & 32 & 18.449 & 10.917 & 1.929 & 0.002 \\
CA-125 & Patient & 36 & 40.873 & 21.067 & 3.511 & \\
& Control & 32 & 17.743 & 15.398 & 2.722 & 0.000 \\
miR-155 & Patient & 36 & 20.749 & 10.616 & 1.769 & \\
& Control & 32 & 10.748 & 6.554 & 1.158 & 0.000 \\
\hline
\end{tabular}

SD, standard deviation; SEM, standard error of the mean.

miRNAs may play a role as oncogenes or tumor-suppressor genes involved in cancer (10). The mechanisms of miRNA expression are still lacking towards our understanding of this type of small endogenetic RNAs since miRNAs frequently target hundreds of mRNAs, and the related regulatory pathways are complex as well. To provide a critical overview of miRNA dysregulation in cancer, scientists must first discuss the methods currently available to review miRNAs in genomic organization, biogenesis and mechanisms of target recognition, and examine how these processes are altered in tumorigenesis (11).

It is now known that miRNAs play major roles in the regulation of gene expression during organism development (12). Researchers are currently using miRNA expression signatures to classify human cancers and are trying to define miRNA markers that might predict favorable prognosis (13). In the field of lung cancer research, it is probable that the differences in miRNA expression may simply be a surrogate for cytogenetic changes in lung cancers (14). Genome-wide expression profiling of miRNAs was found to be significantly different between primary lung cancers and corresponding noncancerous lung tissues. The microarray data were validated by both solution hybridization detection method and real-time RT-PCR analysis for miRNAs. In this way, miRNAs might prove useful in the diagnosis and treatment of cancer.

During the last decade of the 20th century, the most important oncologically related miRNAs have been identified as the miR-21 and let-7 families (15), which are the main research subjects in the field of cancer, including lung cancer. 
Table V. Area under the ROC curve analysis of CEA, CA-125 and miR-155 expression in the serum samples of the lung adenocarcinoma patients and controls.

Asymptotic $95 \%$ confidence interval

\begin{tabular}{|c|c|c|c|c|c|}
\hline Variables & Area & $\mathrm{SE}^{\mathrm{a}}$ & Asymptotic significance ${ }^{\mathrm{b}}$ & Lower bound & Upper bound \\
\hline CEA & 0.712 & 0.063 & 0.003 & 0.588 & 0.837 \\
\hline CA-125 & 0.816 & 0.053 & 0.000 & 0.712 & 0.919 \\
\hline miR-155 & 0.761 & 0.058 & 0.000 & 0.648 & 0.875 \\
\hline
\end{tabular}

The test result variable(s): CEA, CA-125 has at least one tie between the positive actual state group and the negative actual state group. Statistics may be biased. ${ }^{a}$ Under the non-parametric assumption; ${ }^{b}$ null hypothesis: true area, 0.5. SE, standard error.

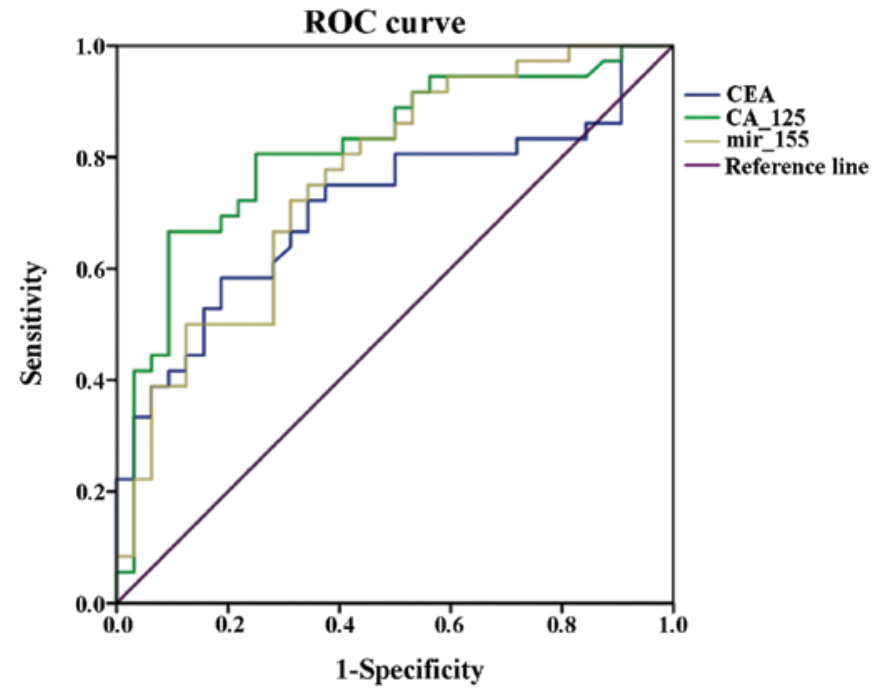

Figure 3. ROC analysis curve of CEA, CA-125 and miR-155 expression in the serum of the lung adenocarcinoma cancer patients and healthy controls.

Researchers have made marked achievements in increasing the knowledge concerning miRNAs. For example, research results on miR-21 expression profiles have demonstrated a link between miR-21 and epidermal growth factor receptor (EGFR) gene mutations. Moreover, high levels of miR-21 expression have been reported in various types of human tumors (16), including but not specifically in lung cancer. Considering this, we aimed to identify more specific options to assistant lung cancer detection and diagnosis. Another reason why we decided to study miR-155 was that previous reports showed that a high level of human miR-155 expression was significantly an unfavorable prognostic factor independently associated with lung cancer progression (17), which indicates that miR-155 could be a strong potential regulator in NSCLC.

Previous research results on miR-155 were from patient tissues and others were based on in vitro study of lung cancer cell lines (3). Moreover, different non-invasive methods were developed to identify the expression of miR-155, such as patient sputum screening inspection (18). For clinical application purposes, of all the above approaches, either they are not reflections of real-time activities of miRNAs in human bodies, or the pathologic samples are not easy and convenient to be collected. In addition, it is barely possible to obtain stable and reliable results in sputum. In order to make an effort in improving these issues, we observed miR-155 levels in serum and chose miR-21 as a positive control. We then aimed to ascertain the role of miR-155 in lung adenocarcinoma occurrence and progression.

As shown in Table III, we compared the levels of miR-155 and miR-21 expression between control and patient groups by defining the level of miR-155 control as the standard reference. Based on this, miR-155 patient was overexpressed $(\mathrm{P}<0.05)$ which was similar to the miR-21 expression in the two groups. The difference in miR-21 levels between the two groups was more

Table VI. Diagnostic index of CEA, CA-125 and miR-155 based on ROC analysis results.

\begin{tabular}{lcccccr}
\hline Inspected parameter & OOP & Sensitivity & Specificity & +LR & -LR & YI \\
\hline CEA $(\mathrm{ng} / \mathrm{ml})$ & 26.532 & 0.583 & 0.812 & 3.101 & 0.514 & 0.395 \\
CA-125 $(\mathrm{U} / \mathrm{ml})$ & 32.386 & 0.667 & 0.906 & 7.096 & 0.368 & 0.573 \\
miR-155 (CT value) & 14.880 & 0.722 & 0.687 & 2.308 & 0.405 & 0.409 \\
CEA + CA-125 & $/$ & 0.889 & 0.750 & 3.556 & 0.148 & 0.639 \\
CEA + miR-155 & $/$ & 0.833 & 0.718 & 2.954 & 0.233 & 0.552 \\
CA-125 + miR-155 & $/$ & 0.889 & 0.688 & 2.849 & 0.161 & 0.576 \\
CEA + CA-125 + miR-155 & $/$ & 0.916 & 0.656 & 2.663 & 0.128 & 0.573
\end{tabular}

OOP, optimal operating point; YI, Youden's index; +LR, positive likelihood ratio; -LR, negative likelihood ratio. 
marked than that of miR-155. The results indicated that the use of miR-21 as positive control between patient and control groups was a good choice; in this way the fold-difference was easily discerned. However, further study is still required to estimate whether miR-155 is suitable for use as a potential novel factor in lung adenocarcinoma diagnosis, even after confirming it was highly expressed in the lung cancer patient group.

To better understand the clinical value of miR-155 in diagnosis and prognosis in lung adenocarcinoma, it was compared with classic biomarkers currently used to benefit clinical outcome. We tested CEA, a glycoprotein involved in cell adhesion, which is not usually present in the peripheral blood of healthy adults and the measurement of which is mainly used as a tumor marker as its expression is specific to cancer cells, particularly adenocarcinomas (19). It can, therefore, be used to distinguish between these and other similar cancers or benign diseases (20). Another biomarker we tested was CA-125, which is encoded by the MUC16 gene, which is a member of the mucin family glycoproteins that has been shown to play a role in advanced tumorigenesis and tumor proliferation by several different mechanisms. It has been identified as a tumor marker or biomarker that may be elevated in the peripheral blood of patients with specific types of cancers (21).

While the value of miR- 155 was estimated for the diagnosis of lung adenocarcinoma patients independently and also combined with serum CEA or CA-125 levels, we used a receiver-operating characteristic curve analysis method (22), and several indices were used to identify the efficacy of each combination group.

The index of sensitivity (also called the true positive rate) relates to the ability of a test to identify positive results. While specificity (sometimes called the true negative rate) relates to the ability of a test to identify negative results. The specificity of a test is defined as the proportion of patients that are known not to have the disease who will test negative for it. These two measures are closely related to the concepts of type I and type II errors. A perfect predictor would be described as having $100 \%$ sensitivity and $100 \%$ specificity. However, theoretically any predictor possesses a minimum boundary error. For any test, there is usually a trade-off between the measures. This trade-off can be represented graphically as a receiver operating characteristic curve (23). The OOP (optimal operating point) is determined with the highest of Youden's index (YI), which is defined as 'sensitivity + specificity - 1'. It is also called Youden's J statistic, a statistic that captures the performance of a diagnostic test.

Compared with the diagnostic value of CEA, CA-125 and miR-155 individually, miR-155 reached the highest sensitivity at 0.722 , which was quite appropriate for early detection of the disease. Of all the three studied markers, CA-125 had the best YI, +LR and -LR scores, which indicates that it is a suitable indicator for adenocarcinoma diagnosis. CA-125 is sufficiently reliable for cancer monitoring. Yet, we were not satisfied with the existing biomarker and wished to explore novel ones for lung cancer diagnosis. This was because confirmation of this disease at a very early stage is greatly beneficial to improving the outcome and prognosis for patients. In this case, sensitivity of a biomarker shows great importance for improving therapeutic strategy. We were interested in identifying the effectiveness of the combination of CEA, CA-125 and miR-155 with each other as different groups. The 'CA-125 + CEA' combination had the best score for YI, while the 'CA-125 + miR-155' combination showed significantly enhanced sensitivity when compared with CA-125. Based on ROC curve analysis, miR-155 had a better AUC than CEA. In addition, relative research has suggested that CEA blood test is not reliable for diagnosing cancer or as a screening test for early detection of cancer. This motivated us to take advantage of a dual test of miR-155 and CA-125 to ameliorate missed diagnosis particularly at the very early stage of lung adenocarcinoma.

In summary, miR-155 is a useful novel tumor marker for lung cancer. It can be stably measured in patient serum with marked differences when compared with health controls. It shows a better specificity than CA-125 and CEA in adenocarcinoma diagnosis. Abnormal high serum concentrations of miR-155 are mainly found in cancers, particularly in NSCLC. Combined testing of miR-155 improves the utility of CA-125 as a tumor marker in lung adenocarcinoma, and using both markers simultaneously increases the tumor marker sensitivity in lung adenocarcinoma diagnosis. Moreover, considering the characteristics of miRNAs, non-coding and short in length, we are able to test its expression in peripheral blood without severe invasion, and more importantly, there is a possibility that we are able to manually modulate miRNA levels easily to cause an alteration in the expression of cancer-related downstream proteins, which are targeted and regulated by the corresponding miRNAs. Currently, the investigation of miRNAs as biomarkers for improving the diagnosis of NSCLC by our research team is just at the onset. We believe that profound effects and benefits provided by miRNAs in disease diagnosis, therapy and prognosis will be achieved in the near future.

\section{References}

1. Nirasawa S, Kobayashi D, Tsuji N, Kuribayashi K and Watanabe N: Diagnostic relevance of overexpressed Nanog gene in early lung cancers. Oncol Rep 22: 587-591, 2009.

2. Duffy MJ: Role of tumor markers in patients with solid cancers: a critical review. Eur J Intern Med 18: 175-184, 2007.

3. Esquela-Kerscher A and Slack FJ: OncomiRs - microRNAs with a role in cancer. Nat Rev Cancer 6: 259-269, 2006.

4. Calin GA and Croce CM: MicroRNA signatures in human cancers. Nat Rev Cancer 6: 857-866, 2006.

5. Soejima M, Tsuchiya Y, Egashira K, Kawano H, Sagawa K and Koda Y: Development and validation of a SYBR Green I-based real-time polymerase chain reaction method for detection of haptoglobin gene deletion in clinical materials. Transfusion 6: 1322-1327, 2010.

6. Alam MS, Mohon AN, Mustafa S, Khan WA, Islam N, Karim MJ, Khanum H, Sullivan DJ Jr and Haque R: Real-time PCR assay and rapid diagnostic tests for the diagnosis of clinically suspected malaria patients in Bangladesh. Malar J 10: 175, 2011.

7. Pfaffl MW: A new mathematical model for relative quantification in real-time RT-PCR. Nucleic Acids Res 9: e45, 2001.

8 Akobeng AK: Understanding diagnostic tests 3: receiver operating characteristic curves. Acta Paediatr 5: 644-647, 2007.

9 Galasso M, Sandhu SK and Volinia S: MicroRNA expression signatures in solid malignancies. Cancer J 3: 238-243, 2012.

10. Calin GA, Sevignani C, Dumitru CD, et al: Human microRNA genes are frequently located at fragile sites and genomic regions involved in cancers. Proc Natl Acad Sci USA 101: 2999-3004, 2004.

11. Farazi TA, Spitzer JI, Morozov P and Tuschl T: miRNAs in human cancer. J Pathol 2: 102-115, 2011.

12. Ambros V: MicroRNA pathways in flies and worms: growth, death, fat, stress, and timing. Cell 113: 673-676, 2003. 
13. Lu J, Getz G, Miska EA, Alvarez-Saavedra E, Lamb J, Peck D, et al: MicroRNA expression profiles classify human cancers. Nature 435: 834-838, 2005.

14 Keller A, Leidinger P, Borries A, Wendschlag A, Wucherpfennig F, Scheffler M, Huwer H, Lenhof HP and Meese E: miRNAs in lung cancer - studying complex fingerprints in patient's blood cells by microarray experiments. BMC Cancer 9: 353-363, 2009.

15. Esquela-Kerscher A, Trang P, Wiggins JF, Patrawala L, Cheng A Ford L, Weidhaas JB, Brown D, Bader AG and Slack FJ: The let-7 microRNA reduces tumor growth in mouse models of lung cancer. Cell Cycle 6: 759-764, 2008.

16. Schetter AJ, Leung SY, Sohn JJ, Zanetti KA, Bowman ED, Yanaihara N, Yuen ST, Chan TL, et al: MicroRNA expression profiles associated with prognosis and therapeutic outcome in colon adenocarcinoma. JAMA 299: 425-436, 2008.

17. Yanaihara N, Caplen N, Bowman E, Seike M, Kumamoto K, Yi M, Stephens RM, Okamoto A, Yokota J, Tanaka T, Calin GA, Liu CG, Croce CM and Harris CC: Unique microRNA molecular profiles in lung cancer diagnosis and prognosis. Cancer Cell 3 . 189-198, 2006.
18. Roa W, Brunet B, Guo L, Amanie J, Fairchild A, Gabos Z, Nijjar T, Scrimger R, Yee D and Xing J: Identification of a new microRNA expression profile as a potential cancer screening tool. Clin Invest Med 2: E124, 2010.

19. Grunnet $M$ and Sorensen JB: Carcinoembryonic antigen (CEA) as tumor marker in lung cancer. Lung Cancer 2: 138-143, 2012.

20. Okamura K, Takayama K, Izumi M, Harada T, Furuyama K and Nakanishi Y: Diagnostic value of CEA and CYFRA 21-1 tumor markers in primary lung cancer. Lung Cancer 1: 45-49, 2013.

21. Li X, Asmitananda T, Gao L, Gai D, Song Z, Zhang Y, Ren H, Yang $\mathrm{T}$, Chen $\mathrm{T}$ and Chen $\mathrm{M}$ : Biomarkers in the lung cancer diagnosis: a clinical perspective. Neoplasma 5: 500-507, 2012.

22. Demler OV, Pencina MJ and D'Agostino RB Sr: Equivalence of improvement in area under ROC curve and linear discriminant analysis coefficient under assumption of normality. Stat Med 12: 1410-1418, 2011.

23. Søreide K: Receiver-operating characteristic curve analysis in diagnostic, prognostic and predictive biomarker research. J Clin Pathol 1: 1-5, 2009. 